\title{
The Role of Epidemiology in Medical Education
}

Most medical educators perceive several areas of weakness in the medical curriculum, topics which should be given more emphasis since students, even at graduation, frequently seem unprepared in those disciplines. (The above statement should not be limited to "medical educators," but should be broadened to include most physicians, whether in academics or practice, since nearly all consider themselves experts in medical education-after all, they have had one. In my experience, they are never shy about telling medical school administrators what is wrong with medical education.) Examples of areas commonly mentioned as needing more emphasis are communication skills, physical diagnosis, medical jurisprudence, ethics, medical economics, nutrition, and preventive medicine. I am certainly sympathetic to all of these considerations, and could present arguments for their importance, especially communication and preventive medicine; however, I should like to make the case for the overriding need for enhanced teaching of epidemiology.

From the College of Medicine, University of Nebraska, Omaha, Nebraska. Address reprint requests to Robert $H$. Waldman, $M D$, Dean, College of Medicine, University of Nebraska, Omaha, NE 68105.
Two often repeated educational encounters from my past should exemplify the problem. For several years in the late 1960s and early 1970s, I participated in small group discussions (pre-GPEP) with first-year medical students, helping them learn some microbiology and immunology. I would ask them to interpret the following data: a child is brought to a physician with a sore throat; after an appropriate history and physical examination, the child is given a penicillin injection. About 48 hours later, the child is feeling much better. When asked the reason for this result, more commonly than not, the answer given by the students was that the penicillin cured the sore throat. (Some of the more "knowledgeable" students would volunteer that the child must have had streptococcal pharyngitis.) Only rarely did a student perceive that nothing could be concluded from the case with respect to etiology or treatment efficacy. The most disappointing aspect was that there seemed to be no correlation between insight into this case and whether the student had been a science major or even whether the student had a graduate degree in one of the biomedical sciences.

More recently, I participated in Morning Report in a Department of Medicine (approximately 1,000 times over a period of several years). My greatest disappointment, guaranteed to start my day poorly, would be to have a 
resident (or all too frequently a faculty member) state: "This patient with lupus cerebritis should be treated with prednisone. Remember Mrs. Jones whom we discussed last month; she got better only after we started prednisone." There are many permutations of this statement-different diagnoses, a variety of therapeutic or diagnostic modalities.

Many of our medical students, residents, colleagues in practice, and even faculty do not seem to understand the scientific method. Cause and effect relationships, or the lack thereof, are not understood. The "my-last-case" syndrome is rampant. The limited scientific usefulness of case reports is not appreciated.

Epidemiology is central to an understanding of research methodology and the interpretation of results, which are, in turn, integral to differentiating a profession from a trade. A good practicing physician must be current with the medical literature, but cannot depend on journal editors to keep erroneous conclusions off their pages. The lack of objectivity in medical literature has been beautifully demonstrated by Tom Chalmers. ${ }^{1,2}$ In addition, journal articles may simply broaden the realm from which to choose "my last case," thus legitimizing the "syndrome." I am convinced that a good grounding in epidemiology and critical thinking will enable our graduates to evaluate medical literature much more accurately.
In recent years, it appears that the public has been exposed to an ever-increasing group of highly questionable therapeutic modalities, such as unproven cancer treatments, dietary approaches, and even methods for selecting the sex of offspring. It is my opinion that the effective response to these "quackeries" is education, not legislation. The medical profession must be more proactive in educating the public regarding these "rip-offs," and in order to do this effectively, health care professionals must clearly understand epidemiological methodologies.

In conclusion, although 1 may be criticized for falling into the trap of doing what I am being critical of others for; ie, making conclusions based on "clinical impressions" rather than good epidemiological data, at least I recognize my mistake. It is my opinion that our faculties in both basic and clinical sciences are not doing an adequate job of teaching the scientific method, and therefore epidemiology should have a more prominent place in the curriculum.

\section{REFERENCES}

I. Chalmers TC: Informed consent, clinical research and the practice of medicine. Trans Am Clin Climatol Assoc 1982; 94:204-211.

2. Chalmers TC, Celano P, Sacks HS, et al: Bias in treatment assignment in controlled clinical trials. N EnglJ Med 1983; 309:1358-1361. 